


\section{DIE GRIECHISCHEN \\ CHRISTLICHEN SCHRIFTSTELLER \\ DER ERSTEN JAHRHUNDERTE}

\section{KOPTISCH-GNOSTISCHE SCHRIFTEN \\ ER ST ER BAND}




\title{
KOPTISCH-GNOSTISCHE SCHRIFTEN
}

\author{
ERSTER BAND \\ Die Pistis Sophia \\ Die beiden Bücher des Jeâ \\ Unbekanntes \\ altgnostisches Werk \\ HERAUSGEGEBEN \\ VON \\ CARL SCHMIDT \\ 4., UM DAS VORWORT ERWEITERTE AUFLAGE \\ HERAUSGEGEBEN \\ VON
}

HANS-MARTIN SCHENKE

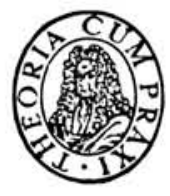

AKADEMIE - VERLAG - B ERL I N 1981 


\section{Herausgegeben von}

Veselin Bałevliev, Ugo Bianchi, Alexander Böhlig, Hans Frbr. v. Campenhausea, Henry Chadwick, Ion Coman, Eligins Dekkers, Gerhard Delling, Jacques Fonteine, Jínos Hermatta, Herbert Hunger, Johannes Irmscher (Verantwortlicher Herausgeber), Robert A. Kraft, Claude Mondéert, Marian Plezis, Harald Riesenfeld, Hans-Martin Bchenke, Wilhelm Schneemelcher, Kurt Treu (Geschäftaführonder Herausgeber),

Ledislar Vidman

Mit Untenstützung des Zentralinstituts für Alte Geschichte and Arch zologie der Akademie der Wisaenschaften der DDR

Erschienen im Akademie-Verlag, DDR - 1080 Berlin, Leipziger Str. 3-4

(C) Akademie-V'erlag 1981

Lizenznummer : $202 \cdot 100 / 222 / 81$

Offretdruck und Bindung: VEB Druckerei „Thomas Müntzer“, 5820 Bad Langensalza

Bestellnummer : 7535248 (2031 6) · LSV 6310

Printed in GDR

DDR 48,- $M$ 